\title{
Refined Estimation of Time-Varying Baseline Errors in Airborne SAR Interferometry
}

\author{
Andreas Reigber, Member, IEEE, Pau Prats, Student Member, IEEE, and Jordi J. Mallorqui, Member, IEEE
}

\begin{abstract}
The processing of airborne synthetic aperture radar (SAR) data requires a precise compensation of the deviations of the platform movement from a straight line. This is usually carried out by recording the trajectory with a high-precision navigation system and correcting them during SAR focusing. However, due to the lack of accuracy in current navigation systems, residual motion errors persist in the images. Such residual motion errors are mainly noticeable in repeat-pass systems, where they are causing timevarying baseline errors, visible as artefacts in the derived phase maps. In this letter, a refined method for the estimation of timevarying baseline errors is presented. An improved multisquint processing approach is used for obtaining robust estimates of higher order baseline errors over the entire scene, even if parts of the scene are heavily decorrelated. In a subsequent step, the proposed method incorporates an external digital elevation model for detection of linear and constant components of the baseline error along azimuth. Calibration targets in the scene are not necessary.
\end{abstract}

Index Terms-Baseline refinement, calibration, interferometry, motion compensation, repeat-pass, synthetic aperture radar (SAR).

\section{INTRODUCTION}

A IRBORNE synthetic aperture radar (SAR) systems usually record the platform movement to later carry out motion compensation during data processing. However, any motion compensation approach is restricted by the quality of the sensor's navigation system, which is nowadays typically limited to a precision of about $1-5 \mathrm{~cm}$. Uncompensated motion errors cause artefacts in the images, among the most important are geometric distortions and phase errors. If a high-precision navigation system is used, such errors are often very small and can be neglected in most applications.

However, this is not the case for interferometric repeat-pass systems. Residual motion errors of each flight track are independent and introduce an unknown time-varying baseline error. In contrast to single-pass systems, residual phase errors caused by this unknown baseline variation do not cancel out during interferogram generation. Even in case of a high-precision navi-

Manuscript received April 14, 2005; revised August 2, 2005. This work was supported in part by the Spanish-German Integrated Action, financed by the German Academic Exchange Service (DAAD) under Grant D/03/40325 and the Spanish Ministry of Communications and Technology (MCYT) under Grant HA2003-0113 and in part by the Spanish MCYT and FEDER funds under Project TIC2003-04451-C02-01

A. Reigber is with the Berlin University of Technology, Berlin 10587, Germany (e-mail: anderl@cs.tu-berlin.de).

P. Prats is with the Universitat Politècnica de Catalunya (UPC), Barcelona E-08034, Spain and also with the Universitat Autònoma de Barcelona (UAB), Bellaterra E-08193, Spain (e-mail: pprats@tsc.upc.edu).

J. J. Mallorqui is with the Universitat Politècnica de Catalunya (UPC), Barcelona E-08034, Spain (e-mail: mallorqui@ tsc.upc.edu).

Digital Object Identifier 10.1109/LGRS.2005.860482 gation system this effect may cause significant phase errors in range and azimuth direction, in principle corresponding to the projection of the time-varying baseline error onto the radar line of sight (LOS).

Recently, there have been some efforts to estimate time varying baseline errors from the processed InSAR data itself and to correct them in a postprocessing step [1]-[4]. However, all of these methods have certain limitations. The methods described in [2] and [3] fully account for the range-dependency of residual phase errors but are unstable in case of low coherence. In contrast, one of the solutions presented in [4] provides only a one-dimensional solution but is very stable in case of decorrelated data sets. Common to all methods is a critical integration step, which relies on high-quality estimates of the derivatives of the residual phase errors.

This letter proposes a refined method for the estimation of time-varying baseline errors that uses the same principle as the two prior methods, but combines the advantages of both in a single algorithm. In Section II-B, an enhanced multisquint processing approach, similar to the one described in [4], is presented. This algorithm provides robust estimates of the derivative of the baseline errors over the entire image, even if some parts of the scene are completely decorrelated. As described in Section II-C, a complete two-dimensional solution can be calculated by a least-square-based estimation, allowing to correctly take into account the variation of phase errors along azimuth and range direction. In a subsequent step, presented in Section II-D, the refined method incorporates an external DEM for the detection of linear components of the baseline error along azimuth, as well as constant baseline offsets. Finally, in Section III, the new algorithm is exemplarily applied on an airborne L-band repeat-pass scene acquired by the German Aerospace Center (DLR) experimental airborne SAR E-SAR.

\section{Estimation OF TIME-VARYING BASELINE ERRORS}

\section{A. Standard Multisquint Processing}

A full-resolution SAR image $S$ is composed of signal contributions with squint angles of a certain range, defined by the length of the processed synthetic aperture and the mean squint angle of the imaging geometry. Out of a full-resolution SAR image with azimuth bandwidth $W$, several subaperture images $S_{i}$, with reduced bandwidth and different mean squint angles, can be formed. Without loss of generality, in the following a mean squint angle $\beta$ of zero will be assumed for the full-resolution SAR image.

Interesting in the context of this letter is the effect of motion errors in the individual subaperture images. As long as the processing is performed in zero-Doppler geometry, the ac- 


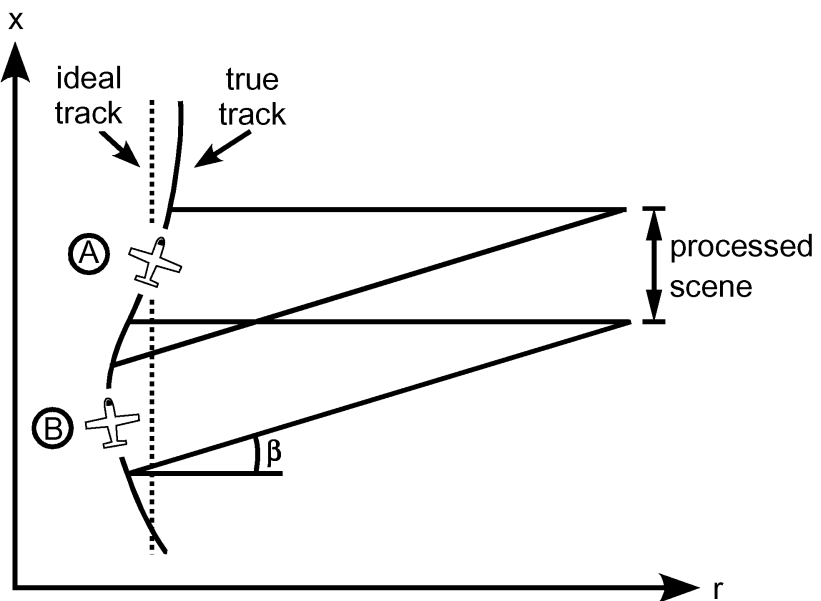

Fig. 1. Standard subaperture processing. Case A: processing with zero squint. Case B: processing with squint $\beta$. A different part of the residual motion error is mapped onto the processed region.

tual image information remains at the same place for all processed subapertures. However, residual phase errors and the corresponding geometrical distortions are not identical, as an aperture, shifted in azimuth by

$$
\Delta x_{i}=r \tan \beta_{i}
$$

relative to the one of the $\beta=0$ case was used for processing, with $r$ denoting the range, and $\beta_{i}$ the mean processing squint of the subaperture. As depicted in Fig. 1, different parts of the uncompensated motion error are mapped onto the same part of the SAR image.

A possibility of estimating residual motion errors would be to process a SAR image twice with different squint angles. The phase difference between the two subapertures should correspond to the difference of the residual motion error, i.e., to the local derivative of the error. Integrating it leads to the residual error itself. However, this approach works only for point-like targets; in case of distributed targets, different part of the ground reflectivity are present in the subapertures, which causes complete decorrelation.

Using interferometry, this problem can be solved: the formation of the interferogram eliminates the influence of the targets reflectivity. Certainly, information about motion errors in the individual tracks are lost and only a time-varying baseline error can be estimated. This approach requires to split both image spectra into positive and negative Doppler-frequencies and to form a differential interferogram between the two subaperture interferograms [5]. Assuming no prior azimuthal coregistration of the image pair, an estimation of the derivative of the baseline error $E$ can be obtained on a pixel-by-pixel basis by [1], [2]

$$
\frac{\partial}{\partial x} E=\frac{v_{0}}{2 \pi r \Delta f} \arg \left\{\left(S_{1}^{1} S_{1}^{2 *}\right)\left(S_{2}^{1} S_{2}^{2 *}\right)^{*}\right\}
$$

with $v_{0}$ denoting the forward velocity, $\Delta f$ the spectral separation of the subapertures, $S_{1}^{1}$ and $S_{1}^{2}$ subaperture 1 of image 1 and 2, and $S_{2}^{1}$ and $S_{2}^{2}$ subaperture 2 of image 1 and 2, respectively. It has to be noted, that, strictly seen, there are additional phase components in (2) which are not related to baseline errors. Also large terrain deformations or velocity errors might cause azimuthal phase effects. Usually their influence is very small;

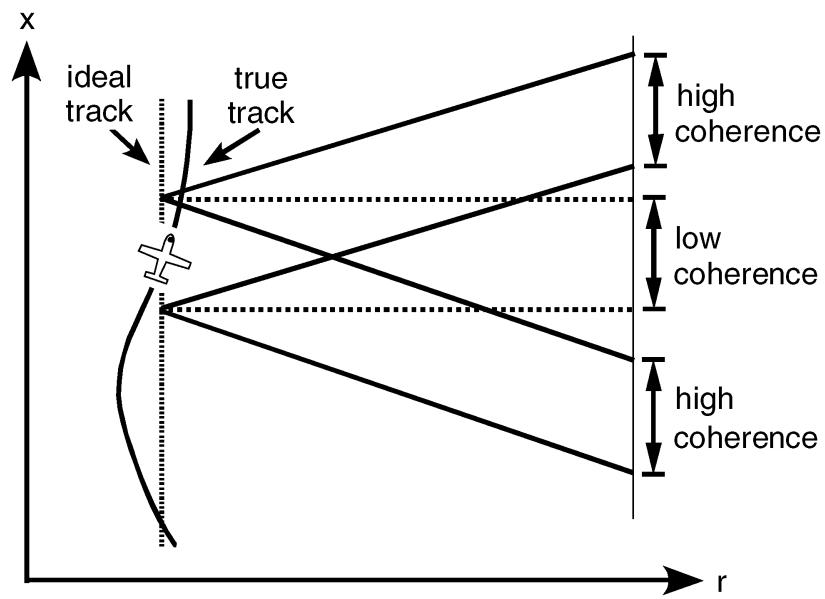

Fig. 2. Enhanced multisquint processing. The baseline error at a low-coherent azimuth position can still be measured as long as it is surrounded by higher coherent regions.

in the following such residual terms will be neglected. However, significant phase errors are caused by crosstalk between topography and motion compensation [10], [11]. which have to be corrected before carrying out (2).

\section{B. Enhanced Multisquint Processing}

A major problem in the approach of Section II is the presence of decorrelated regions, in which no estimation can be performed. A better approach is to use more than two subapertures with smaller bandwidth. Splitting the image into $K$ subapertures of bandwidth $W_{\text {sub }}$, separated by $\Delta f_{\text {sub }}, K-1$ spectral diversity phases $\Phi_{i}$ can be calculated between subaperture $S_{i}^{1,2}$ and subaperture $S_{i+1}^{1,2}$.

$$
\Phi_{i}=\arg \left\{\left(S_{i}^{1} S_{i}^{2 *}\right)\left(S_{i+1}^{1} S_{i+1}^{2 *}\right)^{*}\right\} .
$$

Each $\Phi_{i}$ is proportional to the derivative of the baseline error, but, due to the smaller spectral separation of the subapertures, less accurate than the normal spectral diversity solution [5]. Additionally, $\Phi_{i}$ appears shifted by $r \tan \beta_{i, i+1}$ in azimuth (1) compared to a zero squint solution, with $\beta_{i, i+1}=\left(\beta_{i}+\beta_{i+1}\right) / 2$. Phase wrapping effects can be avoided by selecting subapertures with sufficient small spectral separation [5]. Forming a weighted coherent average of all available $\Phi_{i}$, an improved solution can be obtained

$$
\frac{\partial}{\partial x} E=\frac{v_{0}}{2 \pi r \Delta f_{\text {sub }}} \arg \left\{\sum_{i=1}^{K-1} \mathcal{G}_{i}\left(\left|\gamma_{i}\right| \exp \left(i \Phi_{i}\right)\right)\right\}
$$

with $\mathcal{G}_{i}(\ldots)$ denoting the shift operation in azimuth by $r \tan \beta_{i, i+1}$ and $\left|\gamma_{i}\right|$ the absolute value of the complex interferometric coherence of $\Phi_{i}$. This approach makes it possible to limit the negative influence of low coherent areas. As illustrated in Fig. 2, the error of a certain part of the sensor trajectory is contained in every subaperture. Each one maps the error onto different areas of the imaged scene with possibly different coherence. Consequently, it might be possible to measure the baseline error over decorrelated areas by using information coming from $\Phi_{i}$ with different squint angles. This assumption is valid as long as within a distance smaller than the total length of the synthetic aperture some coherent region is existing. As 

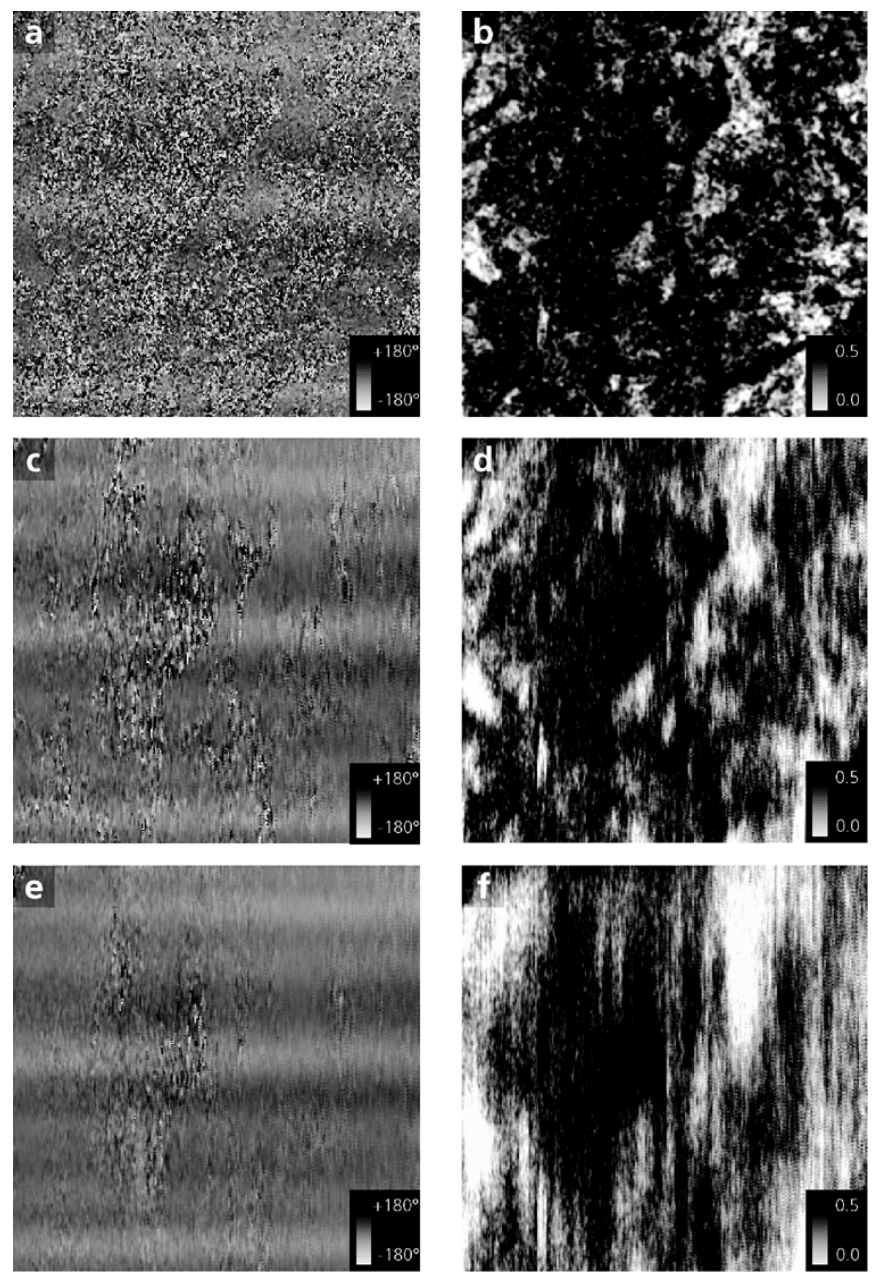

Fig. 3. Enlargement of high-coherent regions by enhanced multisquint processing. (Left column) Estimated $\partial E / \partial x$. (Right column) Estimated weights W. (Top) After processing only the first subaperture. (Middle) After processing one third of the bandwidth. (Bottom) Final solution.

illustrated in Fig. 3, decorrelated regions in the first $\mathcal{G}_{1}(\ldots)$ shrink step by step while adding new $\mathcal{G}_{i}(\ldots)$, weighted by their individual $\left|\gamma_{i}\right|$.

A sufficiently large subaperture separation ensures a high sensitivity to motion errors [5], while short subapertures allow a better estimation of high-frequent errors [6]. The optimal choice of subaperture bandwidth and separation in (4) depends on both the coherence and the amount of residual motion errors and has not been statistically derived yet. Empirically it was found that about five to seven nonoverlapping subapertures out of the full bandwidth seem to be a good compromise at L-band.

\section{Model-Based Integration}

The enhanced solution of (4) represents the derivative in azimuth of the time-variant baseline error $E$ in LOS direction. Apart from its azimuth dependency, $E$ is varying with range, too. The reason is the change of the LOS direction from near range to far range. To obtain the baseline error itself, it is necessary to integrate $\partial E / \partial x$ along azimuth for each range distance [2], [6]. This integration is a critical step, since decorrelated sections of $\partial E / \partial x$ in some of the ranges might prevent a proper solution for these ranges.
As shown in Section II-B, the influence of small decorrelated regions can be eliminated by enhanced multisquint processing. However, it cannot be expected that it is possible to blind out all decorrelated sections for all range distances, particularly when low-coherent data are analyzed. In this case, a robust modelbased integration approach is preferable. The derivative of the baseline error in LOS for range-bin $n$ at a given azimuth position can be calculated ${ }^{1}$ from the derivative in horizontal direction $\partial E_{y} / \partial x$ and the derivative in vertical direction $\partial E_{z} / \partial x$

$$
\frac{\partial}{\partial x} E_{n}=\frac{\partial}{\partial x} E_{z} \cos \theta_{n} \pm \frac{\partial}{\partial x} E_{y} \sin \theta_{n}
$$

using the local off-nadir angle $\theta_{n}$, calculated using an external DEM (see Section II-D). As $\theta_{n}$ are known for all $N$ range-bins of the scene, the baseline error in LOS direction of a given range-line depends only on two free parameters, $\partial E_{y} / \partial x$ and $\partial E_{z} / \partial x$. They can be estimated from only two individual coherent pixels within the range-line. Once they are known, it is possible to integrate them individually along azimuth and to calculate $E$ for the entire scene, i.e., even for completely decorrelated regions.

In most cases the problem is strongly overdetermined, as very likely there are more than two coherent pixels existing in a range line. This holds in particular when the enhanced multisquint processing of Section II-B was applied, which enlarges coherent regions close to the length of the synthetic aperture. In the overdetermined case, a least squares solution can be obtained using

$$
e_{y z}=\left(\mathbf{A}^{T} \mathbf{A}\right)^{-1} \mathbf{A}^{T} e_{\mathrm{los}}
$$

with

$$
\mathbf{A}=\left[\begin{array}{cc} 
\pm \sin \theta_{1} & \cos \theta_{1} \\
\vdots & \vdots \\
\pm \sin \theta_{N} & \cos \theta_{N}
\end{array}\right] \quad e_{y z}=\left[\begin{array}{c}
\frac{\partial E_{y}}{\partial x} \\
\frac{\partial E_{z}}{\partial x}
\end{array}\right]
$$

from the vector formed by the LOS estimations $e_{\mathrm{los}}=$ $\left[\partial E_{1} / \partial x, \ldots, \partial E_{N} / \partial x\right]$ of an entire range line.

Equation (6) does not yet differentiate between high-coherent and low-coherent pixels. To achieve this, a weighted least squares estimation (WLS)

$$
e_{y z}=\left(\mathbf{A}^{T} \mathbf{W A}\right)^{-1} \mathbf{A}^{T} \mathbf{W} e_{\mathrm{los}}
$$

can be used. W denotes a $N \times N$ weighting matrix, which has, in the case of uncorrelated noise, the form [7]

$$
\mathbf{W}=\operatorname{diag}\left\{\frac{1}{\sigma_{1}^{2}}, \frac{1}{\sigma_{2}^{2}}, \ldots, \frac{1}{\sigma_{N}^{2}}\right\}
$$

with $\sigma_{n}$ denoting the standard deviation of the signal at range-bin $n$. Reasonable values for $\sigma_{n}$ can easily be obtained from the interferometric coherence [8]

$$
\sigma=\sqrt{\frac{1-|\gamma|^{2}}{2 L|\gamma|^{2}}} \quad \text { with } \quad \gamma=\frac{1}{K} \sum_{i=1}^{K-1} \gamma_{i}
$$

and $L$ denoting the number of looks. This means that for computing the standard deviation, the mean value $\gamma$ of the individual complex subaperture coherences is used. This ensures that the

\footnotetext{
${ }^{1}$ The sign in (5) depends on the look direction of the sensor (left or right).
} 
enlarged high-coherent regions are correctly taken into account. Additionally, it is helpful to define a coherence threshold (e.g., 0.2 ), under which $1 / \sigma^{2}$ is set to zero.

The result of the inversion of (7) are robust estimates of $\partial E_{y} / \partial x$ and $\partial E_{z} / \partial x$. From few coherent pixels the inversion over the entire scene can be performed. Only two coherent pixels with significantly different range are required for every azimuth bin, with the advantage that prior to the inversion, coherent information of single pixels is spread along azimuth during the enhanced multisquint processing. For azimuth positions with less than two valid pixels, interpolation is required before the integration along azimuth is performed.

\section{Estimation of Constant and Linear Baseline Errors}

One remaining problem during integration of $\partial E_{y}$ and $\partial E_{z}$ are the unknown integration constants. They represent an unknown constant baseline error, which can principally not be estimated by the proposed approach. Its main effect in the SAR interferogram are nonlinear residual fringes in range direction. Another problem is the correct estimation of linear baseline errors. In case of a small global misregistration between the images in azimuth, the estimates of (4) are biased by a constant offset, which is not caused by a baseline error. The consequence is that, after integration, an erroneous linear component appears in the estimated baseline errors, basically provoking a linear fringe pattern in azimuth. Therefore, it is advisable to subtract the mean before integration of $\partial E_{y} / \partial x$ and $\partial E_{z} / \partial x$, leaving the linear baseline error as an unknown.

As both components cannot be estimated from the data alone, a different approach has to be taken. A simple possibility is to use an external digital elevation model (DEM), like for example the one acquired during the Shuttle Radar Topography Mission (SRTM) (see http://www.jpl.nasa.gov/srtm/), to compare the final fringe pattern after compensation of higher order baseline errors with synthetic fringes generated from the DEM [9]. The spatial resolution of the external DEM is of low importance, as it is only desired to estimate 4 parameters of the baseline error, the two constant and the two linear terms of $E_{y}$ and $E_{z}$, respectively.

After correcting higher order terms, as described in the previous two sections, the remaining baseline error $E_{n m}^{\text {res }}$ at range-bin $n$ and azimuth-bin $m$ can be modeled as

$$
E_{n m}^{\mathrm{res}}=\left(E_{z}^{0}+x_{m} E_{z}^{1}\right) \cos \theta_{n} \pm\left(E_{y}^{0}+x_{m} E_{y}^{1}\right) \sin \theta_{n}
$$

with $E^{0}$ and $E^{1}$ denoting the constant and linear terms of the baseline error, and $x_{m}$ the azimuth position at bin $m . E_{n m}^{\text {res }}$ can be directly measured by subtracting a synthetic interferogram, calculated from the external DEM after backgeocoding to slant-range geometry, from the SAR interferogram obtained after applying the refined residual corrections and scaling the result by $\lambda / 4 \pi$. If necessary, the residual interferogram has to be unwrapped before scaling, which is relatively uncritical in this case, since only very few residual fringes can be expected.

As before, an estimation of the four unknown parameters $e_{y z}^{\text {res }}=\left[E_{y}^{0}, E_{y}^{1}, E_{z}^{0}, E_{z}^{1}\right]$ can be obtained by a global WLS optimization

$$
e_{y z}^{\mathrm{res}}=\left(\mathbf{A}^{T} \mathbf{W A}\right)^{-1} \mathbf{A}^{T} \mathbf{W} E^{\mathrm{res}} .
$$

$E^{\text {res }}$ is a vector of the length $N \cdot M$ formed by all pixels of the scaled residual interferogram, with $M$ denoting the number of azimuth-bins. Accordingly, A is a matrix of size $4 \times(N \cdot M)$

$$
\mathbf{A}=\left[\begin{array}{cccc} 
\pm \sin \theta_{1} & \pm x_{1} \sin \theta_{1} & \cos \theta_{1} & x_{1} \cos \theta_{1} \\
\vdots & \vdots & \vdots & \vdots \\
\pm \sin \theta_{N} & \pm x_{1} \sin \theta_{N} & \cos \theta_{N} & x_{1} \cos \theta_{N} \\
\pm \sin \theta_{1} & \pm x_{2} \sin \theta_{1} & \cos \theta_{1} & x_{2} \cos \theta_{1} \\
\vdots & \vdots & \vdots & \vdots \\
\pm \sin \theta_{N} & \pm x_{M} \sin \theta_{N} & \cos \theta_{N} & x_{M} \cos \theta_{N}
\end{array}\right]
$$

while $\mathbf{W}$ is a $N M \times N M$ diagonal weighting matrix, formed analogously to (8). Obviously, the large size of the involved matrices poses a problem. However, since the estimation problem is again strongly overdetermined, it is unproblematic to undersample the residual interferogram by a large factor in order to lower the computational burden when solving (12). Additionally, since $\mathbf{W}$ is diagonal, it is trivial to calculate $\mathbf{A}^{T} \mathbf{W}$ without forming $\mathbf{W}$ itself.

Once $e_{y z}^{\text {res }}$ is known and using (10), a baseline refinement as well as a phase correction for the final interferogram can be calculated.

\section{EXPERIMENTAL RESULTS}

The proposed approach was applied to an L-band interferometric repeat-pass data acquisition of DLR's experimental SAR sensor E-SAR with a baseline of $6.1 \mathrm{~m}$, made in spring 2004 . The test-site is a forested mountainous region in southern Germany $\left(47.75^{\circ} \mathrm{N}, 12.05^{\circ} \mathrm{E}, 3 \times 3 \mathrm{~km}^{2}\right)$, with topographic variations of more than $800 \mathrm{~m}$ across the scene. Because of the strong topography within the scene, an adaptive motion compensation scheme [10] has been used in order to avoid phase errors due to cross-talk between topography and motion errors. The data acquisition interval was 39 days, which causes a relatively low coherence with a mean of $\gamma=0.39$.

The data were processed with an azimuth bandwidth of $150 \mathrm{~Hz}$. No coregistration was performed except compensation of topography related displacements in range direction. During enhanced multisquint processing, a bandwidth of $30 \mathrm{~Hz}$ of the individual subapertures was used, separated from each other by $30 \mathrm{~Hz}$. These parameters give a maximum enlargement of high-coherent areas by about $575 \mathrm{~m}$ along azimuth. Fig. 3 demonstrates this effect. By subsequently adding up more and more subapertures solutions of $\partial E / \partial x$, on the one hand the quality of the estimation of $\partial E / \partial x$ improves, while on the other hand regions of bad estimation due to low coherence shrink step by step.

After obtaining the final estimation of $\partial E / \partial x$, the modelbased integration (7) was applied on each individual range line, using the final matrix of weights as shown in Fig. 3. Additionally, the estimation of linear and constant error terms (11) was performed, based on a SRTM DEM with a grid size of $90 \mathrm{~m}$. For solving (12), an undersampling factor of 8 was used to speed up calculation. From the resulting $e_{y z}$ and $e_{y z}^{\text {res }}$, estimates of $E$ in LOS direction can be obtained for the entire scene. In Fig. 4, the LOS projection at midrange is presented. Fig. 5 shows the original interferogram without corrections and an improved interferogram after reprocessing the slave image with a track modified

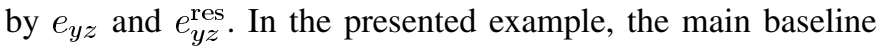




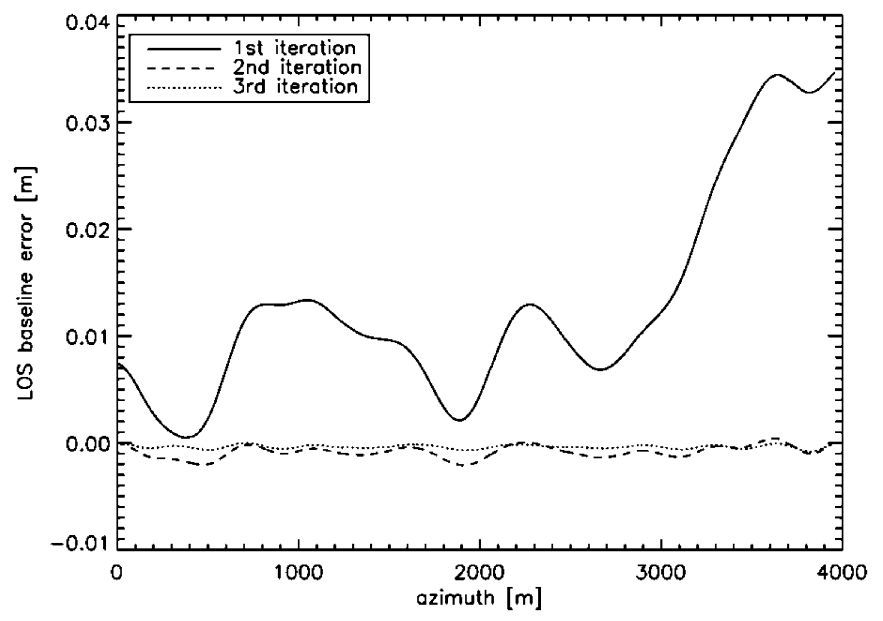

Fig. 4. Baseline error in LOS direction (midrange). Initial estimates and subsequent estimations after repeated application of the proposed method.
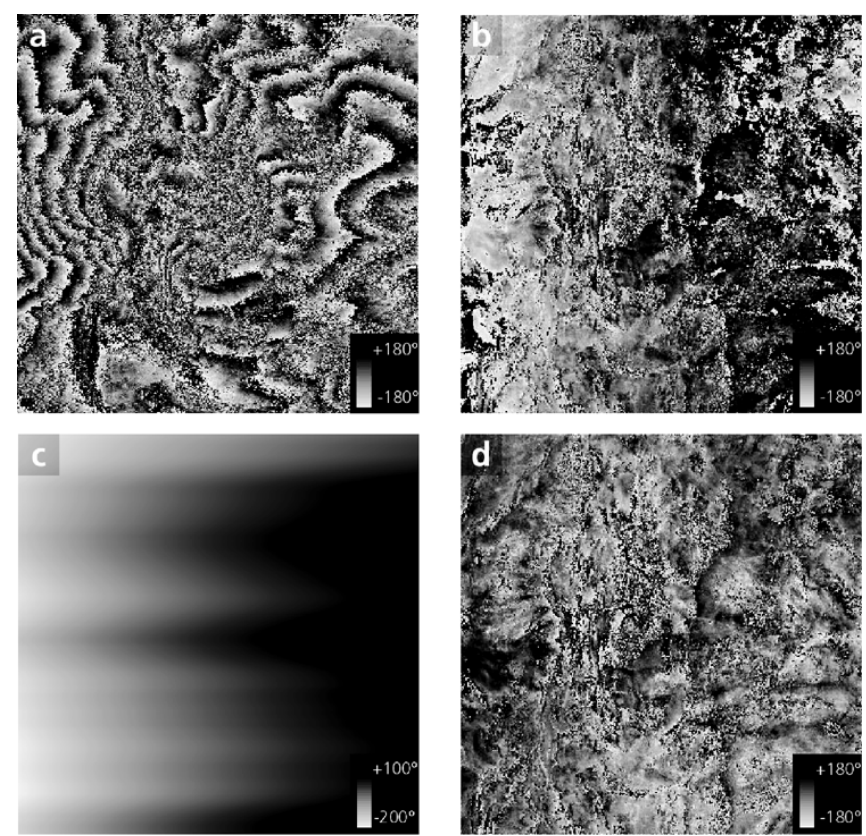

Fig. 5. (a) Initial interferometric phase. (b) Initial interferometric phase after subtraction of topographic component. (c) Phase correction due to estimated baseline variations. (d) Final phase after subtraction of topographic component.

error is a large constant offset of about $13 \mathrm{~cm}$, manifesting itself as a phase ramp across range [Fig. 5(b)]. In contrary, the final residual phase after correction [Fig. 5(d)] seems to be dominated by topographic effects, which could not be removed with the low-resolution SRTM DEM, acquired at a shorter wavelength.

As a check, the proposed method can be applied iteratively on the reprocessed images. In the presented example, subsequent estimations gave very small estimates for $e_{y z}$ and $e_{y z}^{\text {res }}$ in the order of only 1-2 mm (shown in Fig. 4). This value is an indicator for the achievable precision, although for a real evaluation of the proposed algorithm, external data, e.g., acquired by laser tracking, is indispensable.

\section{CONCLUSION}

A refined approach for the estimation of high-frequent baseline errors has been presented. It combines several prior ap- proaches to a robust integrated solution. Even in case of strong decorrelation, the refined approach is capable of detecting small baseline variations and in this way to estimate the corresponding phase errors across the entire scene. Its main disadvantage is the computational burden for forming several subaperture interferograms. In practice, the time needed for baseline refinement is comparable to the time needed for the SAR processing itself.

Apart from scene coherence, the accuracy of the algorithm depends also on the system parameters, as well as on the separation and bandwidth of subapertures. Also other residual effects, in particular large terrain movements in azimuth between the data takes or large velocity errors, can lower the precision of the proposed approach. Topography adaptive motion compensation is crucial for airborne repeat-pass interferometry and no accurate estimation of baseline errors is possible without applying such compensation.

The true accuracy of the presented method is unclear and needs further investigation. As an indication, a convergence to estimates of few millimeters were found in case of E-SAR L-band data. This precision lies significantly above the accuracy of up-to-date navigation systems.

The proposed method can equally be applied to single-pass systems, where errors in the measurement of the lever arms or rotation angles occur. The high scene coherence in this case provides an optimum base for the proposed method.

\section{ACKNOWLEDGMENT}

The authors wish to thank the German Aerospace Center (DLR) for supplying the E-SAR data, and the NASA/SRTM team for providing the DEM.

\section{REFERENCES}

[1] S. N. Madsen and H. A. Zebker, "Imaging radar interferometry," in Manual of Remote Sensing. New York: Wiley, 1998, ch. 6.

[2] A. Reigber, "Correction of residual motion errors in airborne SAR interferometry," Elec. Lett., vol. 37, no. 17, pp. 1083-1084, Aug. 2001.

[3] P. Prats and J. J. Mallorqui, "Estimation of azimuth phase undulations with multisquint processing in airborne interferometric SAR images," IEEE Trans. Geosci. Remote Sens., vol. 41, no. 6, pp. 1530-1533, Jun. 2003.

[4] P. Prats, J. J. Mallorqui, A. Reigber, and A. Broquetas, "Calibration of airborne SAR interferograms using multisquint processed image pairs," in Proc. SPIE, Barcelona, Spain, 2003.

[5] R. Scheiber and A. Moreira, "Coregistration of interferometric SAR images using spectral diversity," IEEE Trans. Geosci. Remote Sens., vol. 38, no. 5, pp. 2179-2191, Sep. 2000.

[6] P. Prats, A. Reigber, and J. J. Mallorqui, "Interpolation-free coregistration and phase-correction of airborne SAR interferograms," IEEE Geosci. Remote Sens. Lett., vol. 1, no. 3, pp. 188-191, Jul. 2004.

[7] A. Sen and M. Srivastava, Regression Analysis: Theory, Methods, and Applications. New York: Springer-Verlag, 1990.

[8] E. Rodriguez and J. M. Martin, "Theory and design of interferometric synthetic aperture radars," Proc. Inst. Elect. Eng. F, vol. 139, no. 2, Apr. 1992.

[9] R. Scheiber, "A three-step phase correction approach for airborne repeat-pass interferometric SAR data," in Proc. IGARSS, vol. 2, 2003, pp. $1190-1192$.

[10] P. Prats, A. Reigber, and J. J. Mallorqui, "Topography-dependent motion compensation for repeat-pass interferometric SAR systems," IEEE Geosci. Remote Sens. Lett., vol. 2, no. 2, pp. 206-210, Apr. 2005.

[11] K. A. C. de Macedo and R. Scheiber, "Precise topography- and aperture-dependent motion compensation for airborne SAR," IEEE Geosci. Remote Sens. Lett., vol. 2, no. 2, pp. 172-176, Apr. 2005. 